\title{
Panbiogeografía y modelos digitales de elevación: un caso de estudio con roedores en la Faja Volcánica Transmexicana ${ }^{1}$
}

\author{
Omar Barrera-Moreno², Tania Escalante ${ }^{3}$ y Gerardo Rodríguez ${ }^{4}$
}

\begin{abstract}
RESUMEN
En este trabajo se propone una adecuación al método panbiogeográfico utilizando modelos digitales de elevación (MDE). Se analizaron las distribuciones de 12 especies de roedores de la provincia biogeográfica Faja Volcánica Transmexicana (FVT) y los resultados se compararon utilizando un modelo tridimensional. Se generaron los trazos individuales de las 12 especies empleando una herramienta automatizada con lo que se identificaron tres trazos generalizados. Por otro lado, sobre la base de un mapa de curvas de nivel, se generó un MDE raster, se calculó un coeficiente de aumento de la distancia en la topología, y los trazos individuales se midieron sobre el MDE. Además, se calculó la longitud de cada trazo individual sobre un MDE vector, donde finalmente se observaron diferencias en la topología de un trazo con respecto al obtenido en el espacio bidimensional. Los restantes once trazos individuales no presentaron cambio alguno, lo cual podría indicar que los trabajos panbiogeográficos realizados a la fecha en dos dimensiones, han representado bien los patrones de los grupos taxonómicos estudiados. Sin embargo, es necesario continuar realizando modificaciones al método para acercarse lo más posible a la realidad, en particular en áreas con alta heterogeneidad altitudinal.
\end{abstract}

Palabras clave: Biogeografía, trazos, 3D, mamíferos, México.

\begin{abstract}
This paper proposes an adequacy to the panbiogeographic method using digital elevation models (DEM). The distributions of 12 species of rodents from the Transmexican Volcanic Belt (TVB) were analyzed, and the results were compared using a three-dimensional model. Individual tracks for the 12 species were draw employing an automated tool which identified three generalized tracks. On the other hand, upon the base of a contour lines map, a raster DEM was built, a rate of increase of distance in the topology was calculated, and the individual tracks were measured on the DEM. Additionally, the length of each individual track was calculated on a vector DEM, where differences in the topology of one track compared to the two-dimensional track were observed. The other eleven tracks did not show any changes, which could means that the actual panbiogeographic analyses in two-dimensions had represented adequately the patterns of the taxonomic groups studied. However, it is necessary to keep making modifications to the method in order to approach as near as possible to the reality, mainly in areas with high altitudinal heterogeneity.
\end{abstract}

Key words: Biogeography, tracks, 3D, mammals, Mexico.

\footnotetext{
1 Artículo recibido el 10 de septiembre de 2009, aceptado el 18 de diciembre de 2009 y corregido el 6 de enero de 2010.

2 Posgrado en Ciencias del Mar y Limnología, Universidad Nacional Autónoma de México (México). E-mail: omarichdien@gmail.com
}

\footnotetext{
3 Museo de Zoología "Alfonso L. Herrera", Universidad Nacional Autónoma de México (México). E-mail: tee@ibiologia.unam.mx

4 Laboratorio de Macroecología, Universidad Nacional Autónoma de México (México). E-mail: gerardo@ecologia.unam.mx
} 
Recientemente se ha propuesto la existencia de una "biogeografía evolutiva" como una ciencia integradora de los métodos biogeográficos históricos (Morrone, 2007; Morrone, 2009). Una de las etapas de un análisis biogeográfico evolutivo involucra el reconocimiento de componentes bióticos a través del uso del método de la panbiogeografía. La panbiogeografía fue propuesta por el botánico Léon Croizat (1958; 1964). Este método consiste en comparar los trazos formados al unir los puntos donde se encuentran distintos taxones en un área determinada y posteriormente encontrar patrones de diferenciación geográfica en el espacio y tiempo (Grehan, 2001). La panbiogeografía enfatiza la dimensión espacial o geográfica de la biodiversidad para permitir una mejor comprensión de los patrones y procesos evolutivos, enfocándose en el papel de las localidades en la historia de la vida (Craw et al., 1999).

Las estructuras que sirven de base al método panbiogeográfico son el trazo individual, el trazo generalizado y el nodo (Rojas, 2004). El análisis panbiogeográfico consiste, en primer lugar, en unir puntos (los registros puntuales de un taxón) en un mapa con el criterio de distancia mínima, formando líneas denominadas trazos individuales, que constituyen las coordenadas primarias de un taxón en un lugar y representan la distribución espacial en la que la evolución del taxón ha tenido y sigue teniendo lugar (Contreras y Eliosa, 2001; Grehan, 2001). En este método inicialmente se localizan las dos localidades más cercanas y se conectan por medio de una línea recta; luego, este par de localidades se conectan con la más cercana a cualquiera de las dos; después, se une la localidad más cercana a cualquiera de las tres, y así sucesivamente hasta terminar con todos los puntos de registro y formar un árbol de tendido mínimo o trazo individual. Los trazos individuales interpretan la geometría espacial como un componente explícito, difiriendo así de los mapas de distribución que engloban las localidades de un taxón mediante un polígono (Morrone, 2004).

Posteriormente, al superponer diferentes trazos individuales se buscan sus coincidencias, obteniendo trazos generalizados que representan patrones de distribución actuales de una biota ancestral ampliamente distribui- da en el pasado, fragmentada por eventos físicos como fenómenos tectónicos, climáticos, cambios en el nivel del mar, entre otros (Craw et al., 1999). En un análisis panbiogeográfico, si se tiene una cantidad representativa de trazos individuales, se pueden corroborar con mayor claridad los trazos generalizados. Cuando diferentes trazos generalizados convergen, se identifican nodos, que indican áreas complejas donde biotas ancestrales tuvieron contacto biótico y/o físico (Contreras y Eliosa, 2001; Grehan, 2001).

Rojas (2004) desarrolló una herramienta informática que automatiza la obtención de los trazos necesarios para el análisis panbiogeográfico, optimizando el tiempo en el que se realiza un estudio, sin importar la complejidad espacial que muestre la nube de puntos que representan las localidades de registro de los taxones (Rojas, 2007). Este autor toma en cuenta la curvatura de la Tierra, por lo que al unir dos puntos referidos con coordenadas geográficas ( $x, y)$ utiliza ecuaciones de trigonometría esférica obteniendo mayor exactitud en la distancia medida. Por otro lado, Liria (2008) realizó otra propuesta sistematizada para realizar análisis panbiogeográficos también basada en herramientas computacionales y de análisis espacial. Más recientemente, Cavalcanti (2009) presentó un programa de cómputo llamado Croizat ${ }^{5}$, diseñado específicamente para realizar análisis panbiogeográficos.

Tradicionalmente, en los análisis panbiogeográficos solo se ha utilizado cartografía en la que la información está representada en dos dimensiones $(x, y)$, que solo toman en cuenta los parámetros de longitud y latitud respectivamente, pero sin considerar el factor de la altitud ( $\mathrm{z}$ ). Esto puede representar un problema metodológico, ya que al unir los registros de un taxón (puntos) en un mapa bidimensional, las distancias calculadas al realizar el trazo individual pueden no ser totalmente correctas, provocando que al comparar los trazos individuales, los trazos generalizados y nodos posiblemente no muestren la realidad de los taxones estudiados. En cambio, si a la cartografía se agregan

\footnotetext{
5 Disponible en http://croizat.sourceforge.net/
} 
los valores de la inclinación o pendiente y la altitud (generando un mapa en tercera dimensión), los árboles de distancia mínima que unen los puntos de registro generarán trazos de una mayor congruencia con la distribución real del taxón.

En un mapa en dos dimensiones, la distancia más corta entre dos puntos se calcula mediante una línea recta. Pero si se considera el relieve que hay entre estos dos mismos puntos, la línea recta deja de serlo y la distancia será mayor a la considerada anteriormente dependiendo de la cantidad de segmentos medidos entre ambos extremos (González-Moreno, 2003; Figura No 1). Un Modelo Digital de Elevación (MDE) es una estructura numérica de datos que permite su tratamiento informático y representa la variable de altitud de un área específica (Felicísimo, 1999). La información en un MDE se genera al tener un par de valores de latitud (y) y longitud (x), que muestran la posición horizontal de un punto de la superficie terrestre, unido a un valor $(\mathrm{z})$ correspondiente de altitud topográfica (García-Estrada y LópezHernández, 2003); estos tres valores son expresados dentro de un sistema de proyección geográfica y en un formato que permite su manejo informático en un Sistema de Información Geográfica (SIG). Según la estructura en que se representen los datos, los MDE se dividen en vectorial y raster (Felicísimo, 1994). Los primeros se basan en entidades u objetos geométricos definidos por las coordenadas de sus nodos y vértices, mientras que el modelo raster está basado en localizaciones espaciales, cada una de las cuales cuenta con un valor de la variable para la unidad elemental de la superficie (Felicísimo, 1999). En ambos modelos la organización de los datos tiene variantes, pero las más utilizadas son la red irregular de triángulos (TIN por sus siglas en inglés) de estructura vectorial, y la

Figura $N^{\circ} 1$

Cálculo bidimensional de la distancia entre dos puntos* y cálculo de la distancia entre los mismos puntos tomando en cuenta el relieve del terreno
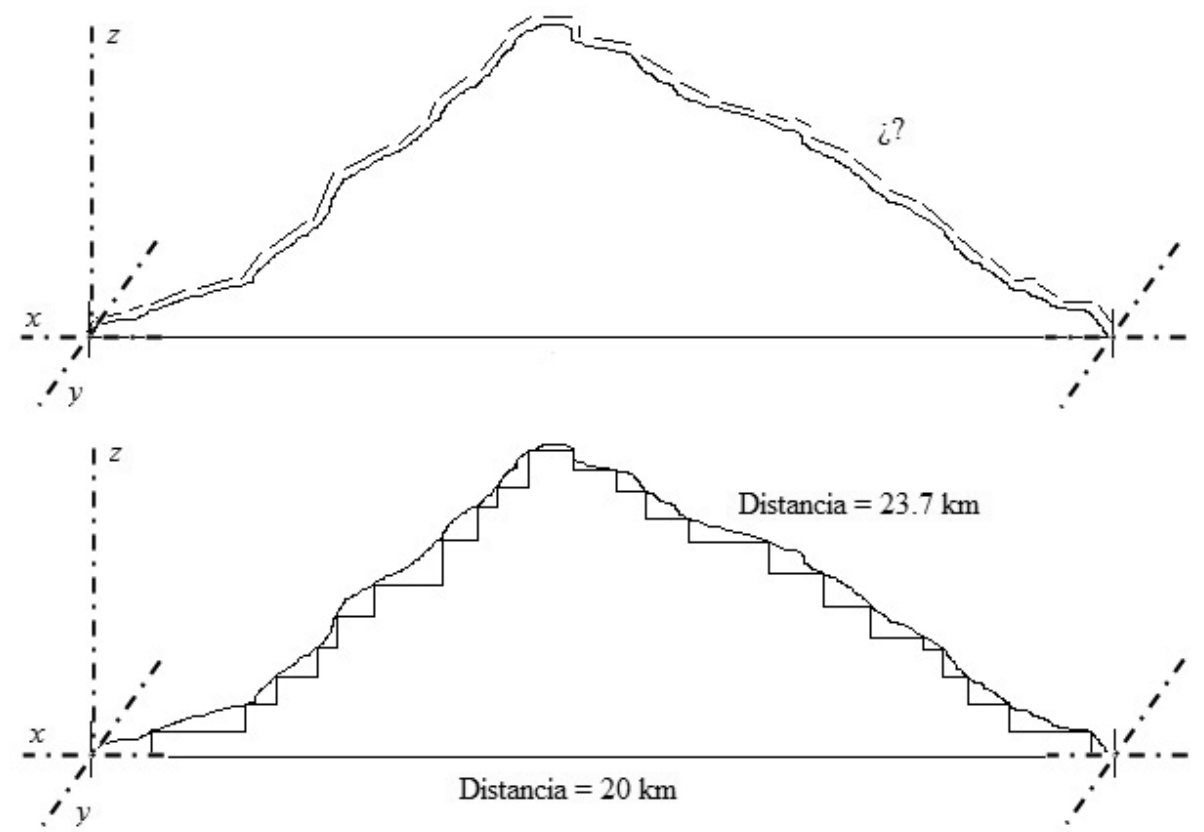

${ }^{*}$ El cual se realiza directamente sobre el eje $y$.

Fuente: Elaboración propia en base a González-Moreno, 2003. 
matriz regular, de estructura raster (Felicísimo, 1999).

El objetivo de este trabajo es mejorar la aplicación del método panbiogeográfico mediante la utilización de Modelos Digitales de Elevación (MDE), estudiando los datos puntuales de 12 especies de roedores de la Faja Volcánica Transmexicana (FVT), con la finalidad de generar hipótesis más adecuadas de los patrones de distribución y los procesos que intervienen en su formación. La comparación entre los trazos en dos y tres dimensiones se realizó mediante: la comparación de la distancia obtenida en los trazos individuales en dos dimensiones y en el MDE; y analizando el cambio en la topología del trazo de dos dimensiones respecto al generado sobre el MDE.

\section{Materiales y métodos}

\section{Área y taxones de estudio}

El estudio de caso se desarrolló en la Faja Volcánica Transmexicana (FVT), localizada en la parte central de México; el polígono utilizado corresponde a los límites propuestos por Morrone (2005). La FVT constituye una expresión fisiográfica de unos $920 \mathrm{~km}^{2}$, predominando altitudes entre los 1.500 y 2.500 m.s.n.m. (Ferrusquía-Villafranca, 1993), pero sobresalen 13 de los 15 picos con más de 3.660 m.s.n.m. que hay en el país (Villa y Cervantes, 2003), y de estos, las tres elevaciones más altas (Pico de Orizaba, Iztaccíhuatl y Popocatépetl). Sus coordenadas extremas aproximadas son entre $18^{\circ} 40^{\prime}-20^{\circ} 55^{\prime}$ latitud $\mathrm{N}$ y $96^{\circ} 30^{\prime}-105^{\circ} 20^{\prime}$ longitud $\mathrm{W}$, atravesando el país de costa a costa (Escalante et al., 2007b). Por sus características, la FVT es una de las regiones biogeográficas y ecológicas más importantes de México (Escalante et al., 2007b).

Debido a la variedad de tipos de vegetación, climas y heterogeneidad del terreno donde se encuentra, la FVT presenta una elevada riqueza de especies de mamíferos y una gran cantidad de endemismos (Fa \& Morales, 1991). Entre las especies exclusivas de la FVT registradas, todas a excepción de Romerolagus diazi (Lagomorpha) y Sorex macrodon (Insectívora), pertenecen al orden
Rodentia (Munguía, 2004; Escalante et al., 2005; Escalante et al., 2007a). Para este estudio se seleccionaron las especies de roedores cuya distribución abarcara casi de forma exclusiva la FVT y contaran con un mínimo de tres puntos de registro. Para esto, se consultó una base de datos con antecedentes de ejemplares de colecciones y literatura (Escalante et al., 2002).

\section{Dos dimensiones}

Utilizando ArcView 3.2 (ESRI, 1999) y la extensión Trazos2004@ (Rojas, 2004), se generaron los trazos individuales de cada especie y los trazos generalizados. Usando la función trazo individual que posee la extensión Trazos 2004@, se unieron mediante la línea de menor distancia los puntos de registro de cada taxón, generando los trazos individuales correspondientes.

Esta herramienta presenta dos etapas (Rojas, 2004): en la primera, crea una matriz de distancias en la que enfrentan las localidades identificadas por un número único. Adicionalmente, se calculan los valores mínimos para cada una de las localidades con respecto a las otras. Después, se crea la tabla espacial para la cobertura del trazo individual; esta contiene dos columnas importantes, una que indica las localidades que se están relacionando, y la otra, las distancias entre las localidades. Estas distancias se relacionan con las mínimas de la tabla matriz inicial, con la que se seleccionan aquellas relaciones entre localidades que cumplen con tener las distancias mínimas. Por último, la herramienta toma como base la columna que contenga los recorridos menores y genera el árbol de tendido mínimo.

Posteriormente, con la función trazo generalizado de la misma extensión, se superpusieron los trazos individuales seleccionados para obtener uno generalizado. Por último, con la herramienta nodos, se seleccionaron los trazos generalizados obtenidos anteriormente y se generaron los nodos que corresponden a la superposición de dos o más de ellos. 


\section{Tres dimensiones}

Debido a que la distribución puntual de algunas especies estudiadas no está totalmente restringida al área de la FVT, para obtener el MDE del área de estudio se utilizó un cua-

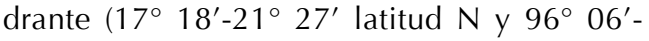
$105^{\circ} 39^{\prime}$ longitud W) que incluyera las curvas de nivel correspondientes al área que abarca la provincia. Las curvas se encuentran cada $200 \mathrm{~m}$ y tienen valores de entre 201 y 5.401 m.s.n.m.

El mapa de curvas de nivel fue proporcionado por el Instituto de Geografía de la UNAM en formato vector y posteriormente se convirtió a raster, utilizando el software Paint Shop Pro 4.12 (JASC Inc., 1996). Usando los algoritmos Reduct_Mnt y Transf._dxf_V2 se generó el MDE con un tamaño final de 856 columnas y 375 líneas. En este MDE de la Faja Volcánica Transmexicana, cada píxel tiene un valor o tamaño de $50 \times 50$ m con su valor correspondiente de altitud. La cobertura de las curvas de nivel que abarcan el área de la FVT toma valores de $200 \mathrm{~m}$ entre cada cota.

Las imágenes de cada trazo individual realizado anteriormente, fueron guardadas en formato de archivo de imagen (.bmp) desde ArcView 3.2 y después se convirtieron a archivos de tipo. raw ya que el software Paint Shop Pro 4.12 (JASC Inc., 1996) así lo requiere. Por otro lado, se construyó un archivo en formato de texto (.txt) de cada especie con la siguiente información obtenida de la imagen: el tamaño de la imagen en píxeles, el tamaño del archivo, los valores en la escala de grises que toma la imagen y el tamaño de la celda o píxel.

Para hacer coincidir la escala de los trazos individuales con la del MDE se usó el algoritmo Extract.exe que corta el archivo del trazo a la misma medida que el del MDE. El resultado es un archivo .raw y un .txt con la información de los valores de cada especie. La imagen .raw se abre en Paint Shop Pro 4.12 para verificar el valor de la línea en la escala de grises, necesaria en el siguiente tratamiento.

Posteriormente, tanto los trazos individuales como el MDE se trataron nuevamente en Paint Shop Pro 4.12, esta vez con el algoritmo
Comp-red3, que calcula las diferencias en distancia (en 2 y 3 dimensiones) con la misma topología de un trazo. El programa genera tres archivos por especie: uno de tipo .raw, uno .txt y una tabla de .xls con los valores de: distancia cenital o 2D (en metros), distancia en 3D (en metros) y desviación estándar.

Con los datos obtenidos de los archivos anteriores y utilizando la siguiente fórmula, se generó un valor que indica cuánto aumentó la distancia del trazo individual de cada especie:

Coeficiente de Aumento de Distancia (CAD) $=$ Dif / Dz x Arctan

Donde:

Dif: diferencia en metros de la distancia del trazo en 3D menos la distancia de trazo en 2D, y

Dz: distancia en 2D o cenital.

EI MDE vector se realizó con la estructura de red irregular de triángulos o TIN, utilizando el software Arc Map 9.2, un subprograma de ArcGIS 9 (ESRI, 2006). El programa usa las mismas curvas de nivel del modelo anterior sin necesidad de convertirlas a otro formato, ya que originalmente están de forma vectorial; solo es necesario georreferenciarlas en coordenadas UTM de tipo World Geodetic System 1984 (WGS84). Debido a que las herramientas de ArcView 3.2 y Paint Shop Pro 4.12 no son suficientes para calcular las distancias entre cada par de puntos considerando el relieve en el MDE raster, se utilizó el MDE de tipo vector (o TIN) en ArcMap 9.2, con el que se pudieron calcular las distancias tridimensionales entre cada par de puntos, obteniendo un valor más cercano a la realidad.

Para obtener en este segundo MDE los valores de cada segmento de los trazos individuales, se convirtieron las coordenadas geográficas de cada punto en coordenadas del sistema UTM. En el MDE vector, las curvas de nivel también se cambiaron al mismo formato. Después se calcularon individualmente las distancias entre cada par de puntos que tuvieran la posibilidad de estar más cercanos con el relieve de por medio y por último se compararon con los puntos unidos en el mapa bidimensional. 


\section{Resultados y discusión}

Se seleccionaron doce especies que bajo los criterios señalados anteriormente fueron consideradas como restringidas a la FVT (Cuadro $\mathrm{N}^{\circ} 1$ ). Se utilizó la clasificación taxonómica de Ramírez-Pulido et al. (2005) para listarlas. En el Cuadro $N^{\circ} 1$ también se muestran las columnas puntos del trazo y puntos en el MDE, que coincidentemente contienen los mismos datos ya que todos los puntos de registro se localizan dentro del MDE ela- borado. Por otro lado, la columna puntos en la FVT muestra la cantidad de registros que solamente se encuentran dentro del polígono de la provincia.

Se obtuvieron doce trazos individuales en dos dimensiones (Figura $N^{\circ} 2 a$ ). El Coeficiente de Aumento de Distancia (CAD) que indica cuánto aumentó el trazo individual de cada especie sobre el MDE con respecto al mismo trazo sobre el mapa bidimensional se muestra en el Cuadro $\mathrm{N}^{\circ} 2$.

\section{Cuadro $\mathrm{N}^{\circ} 1$}

Lista en orden taxonómico de las especies dentro de la FVT y del MDE

\begin{tabular}{|c|l|c|c|c|}
\hline & \multicolumn{1}{|c|}{ Especie } & Puntos del trazo & Puntos en la FVT & Puntos en el MDE \\
\hline 1 & Spermophilus perotensis & 5 & 4 & 5 \\
3 & Reithrodontomys chrysopsis & 40 & 34 & 40 \\
4 & Reithrodontomys hirsutus & 9 & 3 & 9 \\
5 & Meotomodon alstoni & 60 & 50 & 60 \\
6 & Microtus oaxacensis & 11 & 1 & 4 \\
7 & Microtus quasiater & 4 & 4 & 46 \\
8 & Cratogeomys gymnurus & 46 & 10 & 20 \\
9 & Cratogeomys merriami & 20 & 45 & 61 \\
10 & Pappogeomys bulleri & 38 & 20 & 38 \\
11 & Zygogeomys trichopus & 6 & 6 & 6 \\
12 & Liomys spectabilis & 6 & 3 & 6 \\
\hline
\end{tabular}

Fuente: Elaboración propia. 
Panbiogeografía y MOdelos digitales de eleVACIÓN: UN CASO

Figura $\mathrm{N}^{\circ} 2$

Trazos individuales de especies de Rodentia

a) Reithrodontomys hirsutus, b) Spermophilus perotensis

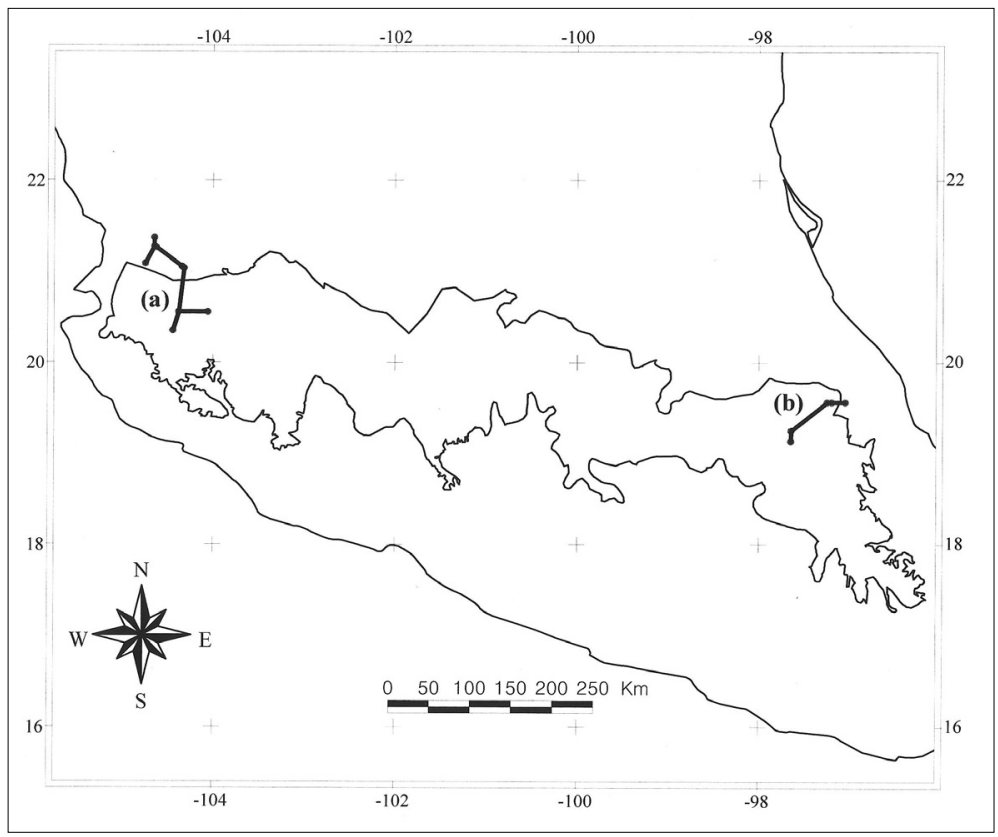

c) Reithrodontomys chrysopsis

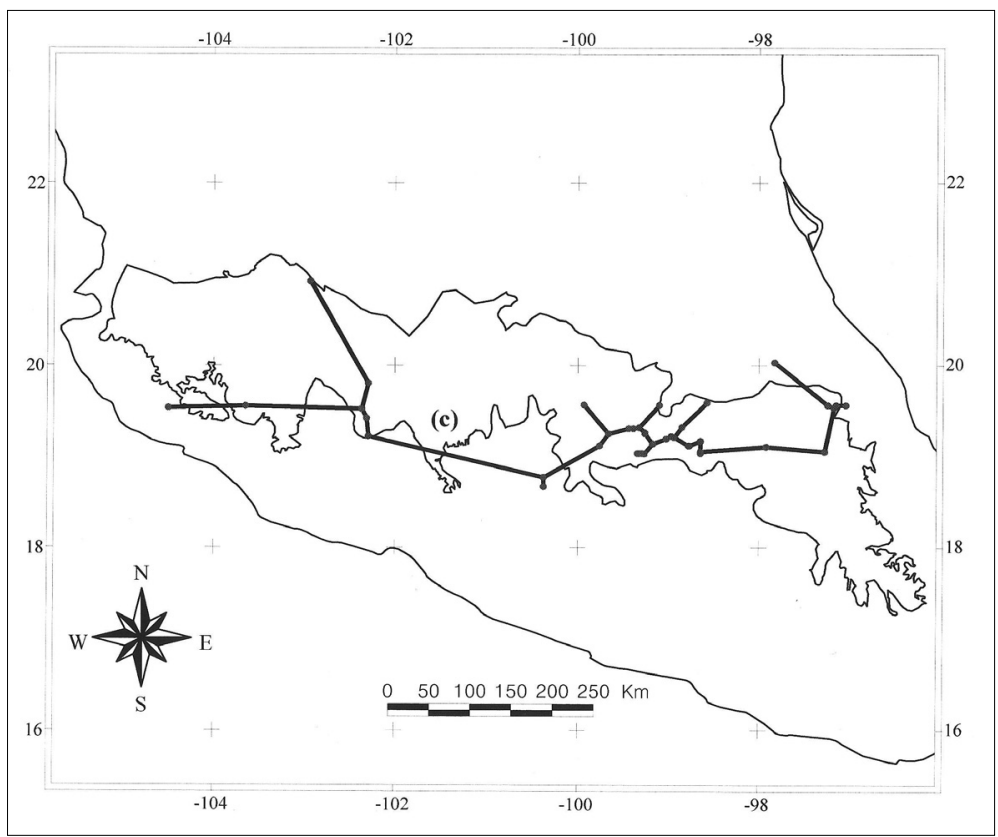


d) Neotomodon alstoni

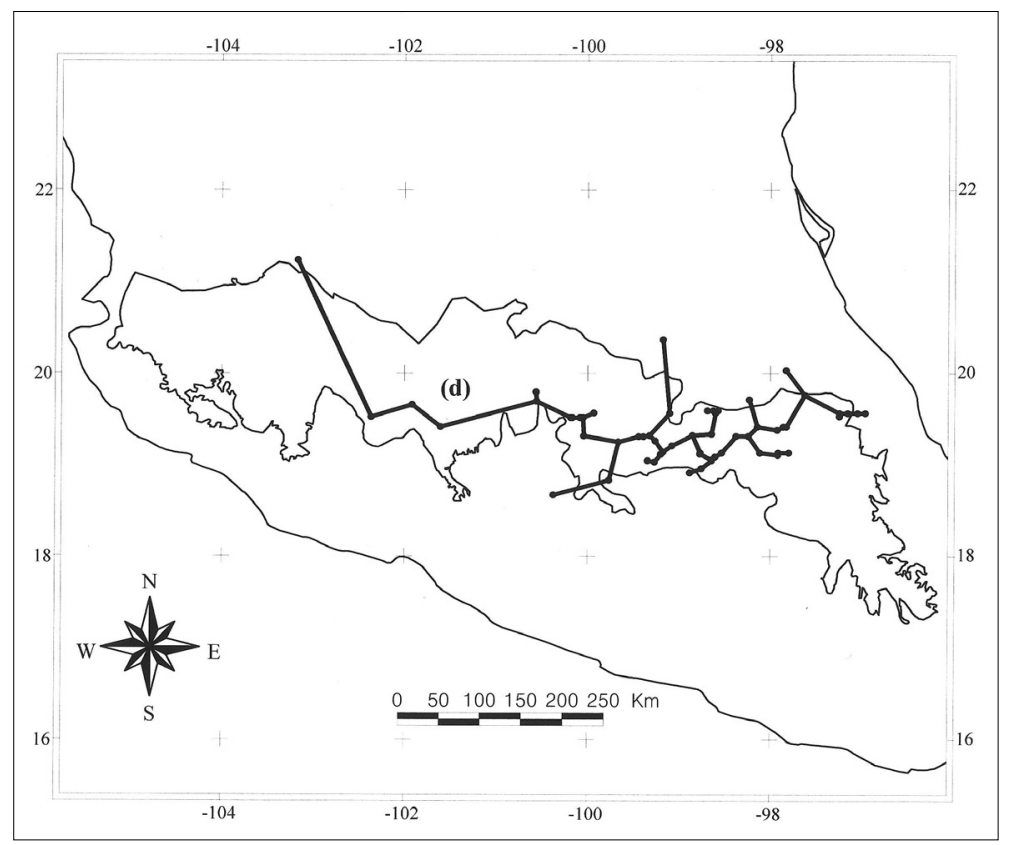

e) Liomys spectabilis, f) Megadontomys thomasi

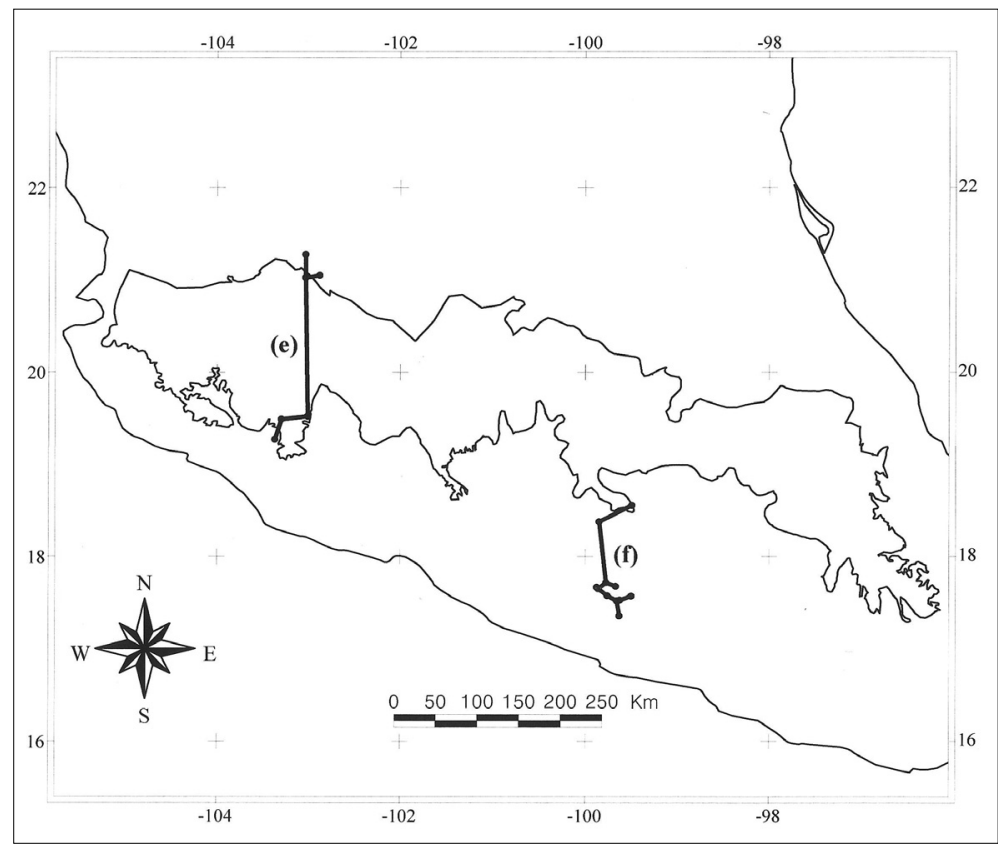


Panbiogeografía y MOdelos digitales de eleVACIÓN: UN CASO

g) Cratogeomys gymnurus, h) Microtus oaxacensis

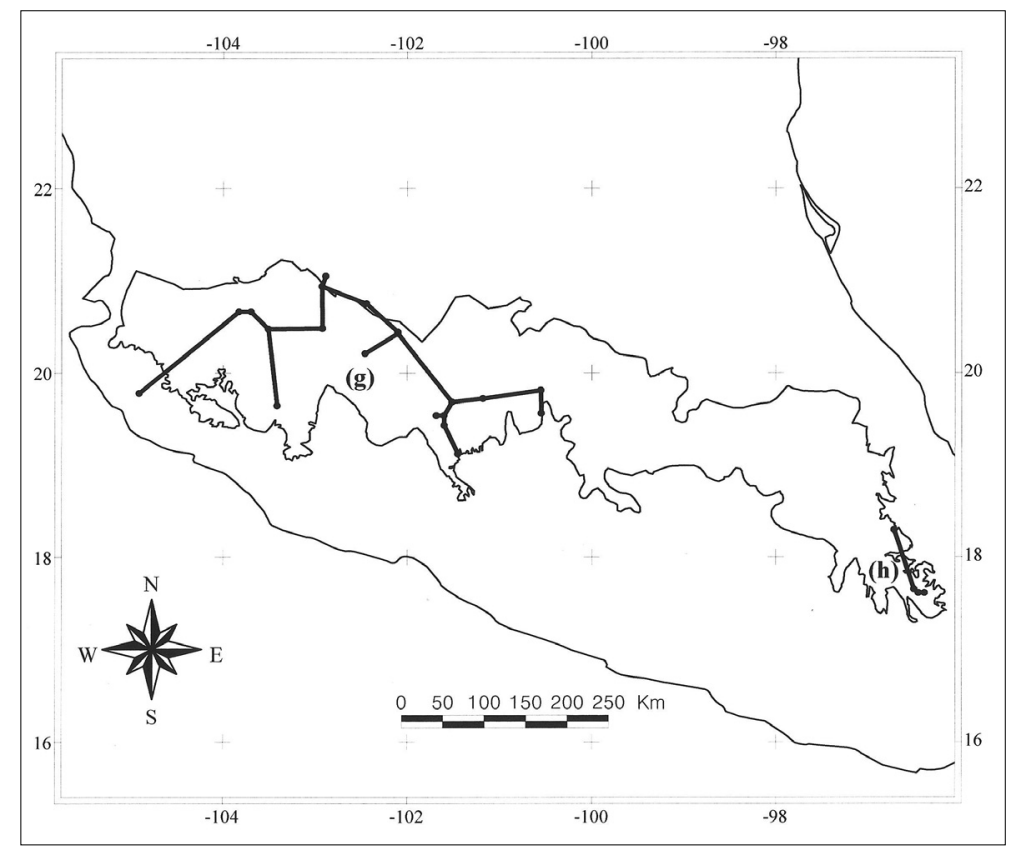

i) Zygogeomys trichopus, j) Microtus quasiater

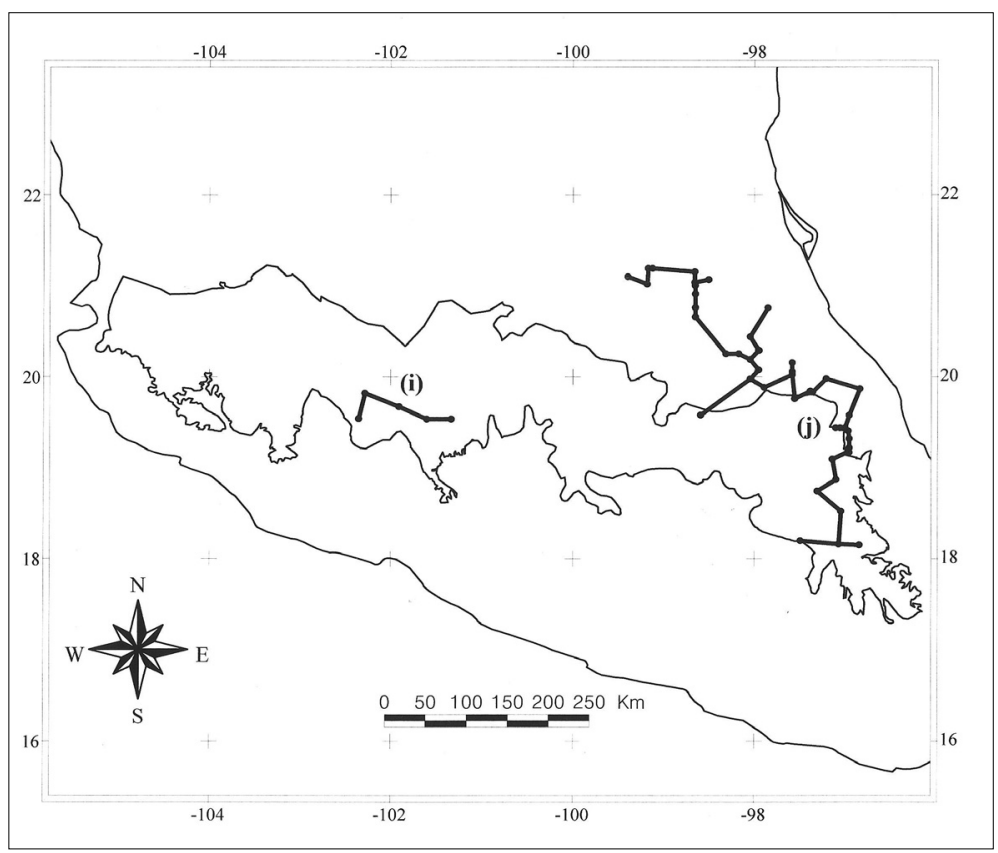


k) Pappogeomys bulleri, I) Cratogeomys merriami

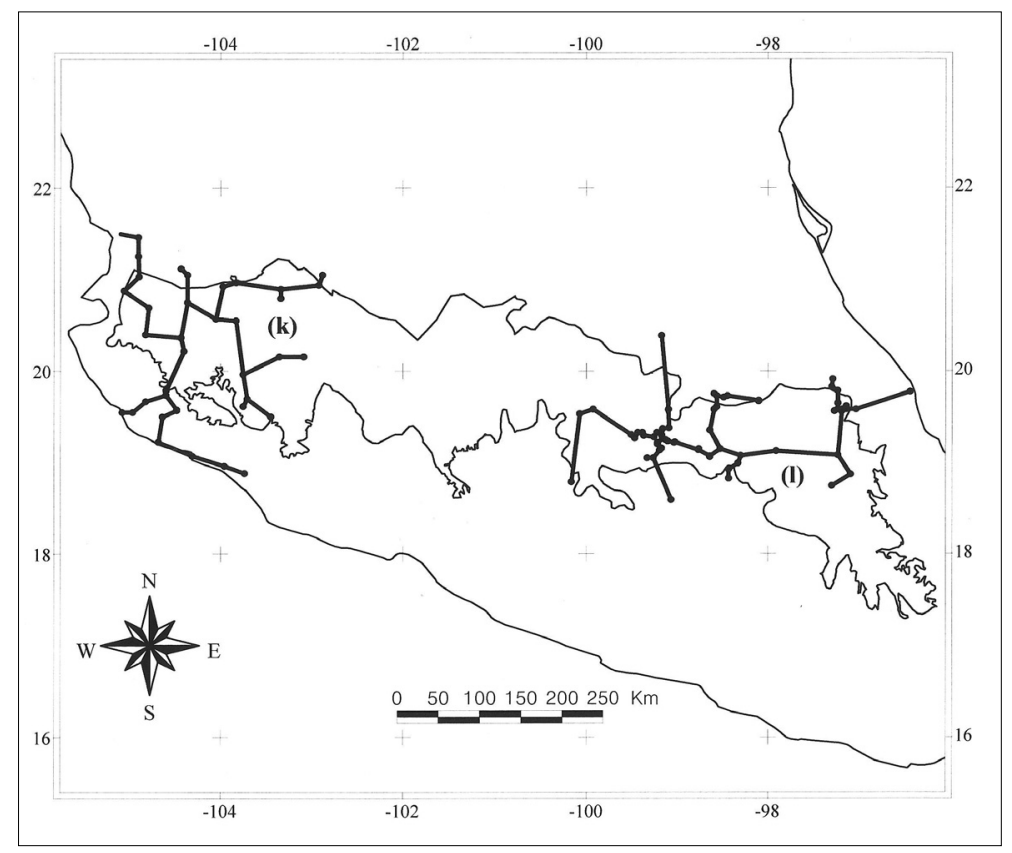

Fuente: Elaboración propia.

Cuadro $\mathrm{N}^{\circ} 2$

Diferencia $(m)$ de la distancia en 2D y 3D de los trazos individuales

\begin{tabular}{|l|c|c|c|c|}
\hline \multicolumn{1}{|c|}{ Nombre (.raw) } & $\begin{array}{c}\text { Distancia cenital o 2D } \\
(\mathrm{m})\end{array}$ & Distancia 3D $(\mathrm{m})$ & Diferencia $(\mathrm{m})$ & $\begin{array}{c}\text { Coeficiente } \\
(\text { CAD })\end{array}$ \\
\hline Spermophilus perotensis & $91.809,1000000$ & $91.866,455006$ & 57,355006 & 0,0357 \\
Reithrodontomys chrysopsis & $1.396 .146,000000$ & $1.397 .488,095389$ & $1.342,095$ & 0,0550 \\
Reithrodontomys hirsutus & $046.000,000000$ & $46.125,612403$ & 125,612403 & 0,1564 \\
Neotomodon alstoni & $1.454 .494,70000$ & $1.456 .129,957751$ & $1.635,257751$ & 0,0644 \\
Megadontomys thomasi & $207.211,600000$ & $207.536,906304$ & 325,306304 & 0,0899 \\
Microtus oaxacensis & $90.776,400000$ & $91.088,515398$ & 312,11539 & 0,1969 \\
Microtus quasiater & $1.763 .210 .645,161$ & $1.764 .615 .119,565$ & $1.404 .474,404$ & 0,0456 \\
Cratogeomys gymnurus & $895.201,40000$ & $895.875,578323$ & 674,1783 & 0,0431 \\
Cratogeomys merriami & $1.062 .025,0000$ & $1.062 .733,876110$ & 708,876 & 0,0382 \\
Pappogeomys bulleri & $1.125 .120,900000$ & $1.126 .028,647975$ & 907,747 & 0,0462 \\
Zygogeomys trichopus & $146.137,400000$ & $146.373,820398$ & 236,4203 & 0,0926 \\
Liomys spectabilis & $260.419,800000$ & $260.598,498815$ & 178,6988 & 0,0393 \\
\hline
\end{tabular}

Fuente: Elaboración propia. 
Por último, al unir los puntos de registro de cada especie sobre el MDE de formato vector, se formaron los nuevos trazos individuales de cada taxón y los trazos generalizados, observándose diferencias solamente en la distancia de un par de segmentos en el trazo de Pappogeomys bulleri. En las once especies restantes, la topología de los trazos no presentó cambios.

A continuación se describen los resultados del análisis para cada especie.

1) Spermophilus perotensis: presentó el menor coeficiente de aumento de la distancia, debido a la cercanía entre los puntos y la poca cantidad de registros. El trazo resultante después de unir los registros sobre el MDE muestra la misma topología que el elaborado en el mapa de dos dimensiones.

2) Reithrodontomys chrysopsis: el CAD para esta especie tuvo un valor de 0,0550, que significó un aumento de $1,34 \mathrm{~km}$ en el trazo en 3D. El trazo elaborado con el MDE no tuvo variaciones en ningún segmento, debido a que los puntos a comparar se situaban dentro de la misma cota de relieve o el más cercano en dos dimensiones era el mismo usando tres dimensiones.

3) Reithrodontomys hirsutus: tuvo el segundo CAD más elevado de todas las especies, pero este aumento en la distancia no significó cambio alguno en la topología del trazo generado sobre el MDE.

4) Neotomodon alstoni: su trazo no tuvo ningún cambio al calcular las distancias entre los puntos con el MDE. Se observó constantemente que entre un par de puntos a medir con un tercero, el relieve fue muy parecido, por lo que el punto más cercano en el mapa bidimensional conservó la característica de seguir siendo el más cercano.

5) Megadontomys thomasi: el valor de su CAD fue 0,0899, y al calcular el trazo individual sobre el MDE, la topología del mismo no sufrió cambios pues aunque el relieve intervino en el aumento de las distancias, los valores no fueron lo sufi- cientemente elevados para modificar la topología del trazo.

6) Microtus oaxacensis: el CAD de su trazo individual fue el mayor de todos, dado que los puntos se localizan en un área pequeña pero con relieve muy heterogéneo. Lo anterior no significó que el trazo individual en tercera dimensión tuviera algún cambio con respecto al trazo original, solo se ratificó que el trazo original fue bien calculado.

7) Microtus quasiater: el CAD mostrado por el trazo individual de esta especie fue relativamente bajo en comparación a las demás especies, pero en su distancia en metros sí mostró una gran diferencia (1.404,47 km más sobre los 1.763.210,64 $\mathrm{km}$ iniciales). Por su parte, el trazo generado sobre el MDE no tuvo cambio alguno en las más de 20 posibilidades calculadas.

8) Cratogeomys gymnurus: el coeficiente generado fue de los más bajos, y el trazo formado a partir de los puntos sobre el MDE no mostró cambios, pues el relieve no interfirió sobre la distancia más corta entre los puntos.

9) Cratogeomys merriami: su CAD fue el segundo más bajo entre todas las especies estudiadas. La distancia lineal se incrementó 0,7 km sobre el MDE, una cantidad mínima si se toma en cuenta que la suma de los segmentos en el trazo original tuvo un valor de $1.062 \mathrm{~km}$. Al unir los puntos con las distancias tridimensionales, el trazo generado mostró exactamente la misma topología que el de dos dimensiones.

10) Pappogeomys bulleri: tuvo un valor del CAD de 0,0462, que significó el incremento de casi $1 \mathrm{~km}$ respecto a la distancia cenital registrada inicialmente. P. bulleri presentó dos cambios en la topología de su trazo individual cuando se calcularon las distancias entre cada punto tomando en cuenta el relieve de la FVT (Figura $N^{\circ} 3$ ), demostrando que esta característica sí puede llegar a intervenir en la formación de los trazos individuales. 
Figura $\mathrm{N}^{\circ} 3$

Trazo individual de $P$. bulleri*

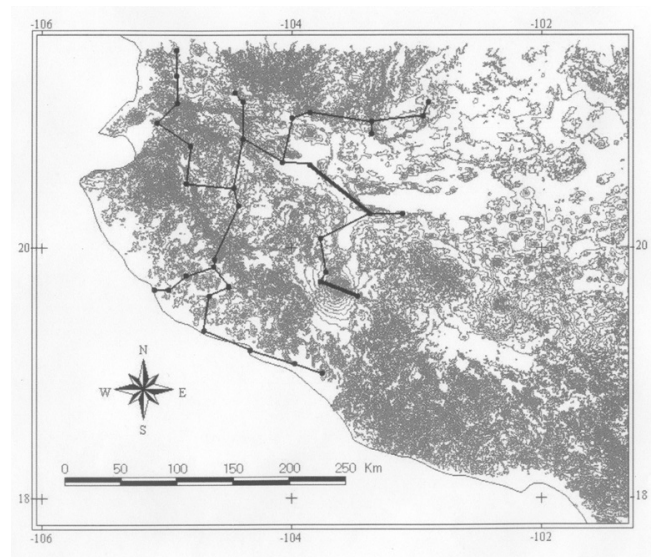

* En líneas más gruesas se señalan los dos segmentos que tuvieron una modificación al calcular las distancias sobre el MDE (se muestran las cotas altitudinales).

Fuente: Elaboración propia.

11) Zygogeomys trichopus: tuvo un CAD de 0,0926, como consecuencia de que los pocos registros que forman el trazo individual se localizan dentro de una zona con relieve prominente; aun así, la cantidad de metros que aumentó la longitud del trazo sobre el relieve fue de solo $236,42 \mathrm{~m}$. Por su parte, el trazo formado sobre el MDE no mostró variaciones con respecto al original, pues se observó que el relieve del área interfiere de la misma manera en un par de puntos medidos, causando que el par más cercano en 2D sea el mismo en 3D.

12) Liomys spectabilis: el CAD de su trazo individual mostró un valor de 0,0393, pero no se observó cambio alguno con respecto a la forma inicial del trazo individual.

\section{Trazos generalizados y nodo}

Se obtuvieron tres trazos generalizados después de comparar todos los trazos individuales (Figura $N^{\circ} 4$ ). Las especies que sustentan el trazo 1 son: Pappogeomys bulleri y Reithrodontomys hirsutus; el trazo 2 lo conforman Cratogeomys gymnurus, C. merriami,
Reithrodontomys chrysopsis, Neotomodon alstoni y Zygogeomys trichopus. Por último, el trazo 3 lo componen: Spermophilus perotensis, Microtus quasiater y $M$. oaxacensis. $M$. thomasi y Liomys spectabilis no intervinieron en la formación de ningún trazo generalizado. Para finalizar, los cambios que tuvieron lugar en un par de segmentos de P. bulleri no generaron modificaciones sobre el trazo generalizado en el que participa, por lo que no fue necesario utilizar los trazos individuales obtenidos sobre el MDE para formar los trazos generalizados.

El trazo generalizado 3 es similar a uno de los obtenidos por Torres-Miranda y LunaVega (2006), quienes analizaron los puntos de registro de 136 especies de helechos, encinos, mamíferos, aves y reptiles. Además, se destaca que los trazos generalizados 2 y 3 coinciden en parte con dos de los obtenidos por Escalante et al. (2004) utilizando 28 taxones de mamíferos pertenecientes a los órdenes Insectívora, Lagomorpha y Rodentia; y con los generados por Álvarez-Mondragón y Morrone (2004) al estudiar 139 especies de aves terrestres de México.

En la única intersección de los trazos generalizados 2 y 3 se formó un nodo (Figura $N^{\circ} 4$ ), lo que representa un área compleja donde biotas ancestrales tuvieron contacto. El nodo coincide de manera casi perfecta con uno de los obtenidos por Escalante et al. (2004), sin embargo, es recomendable corroborar tanto los trazos generalizados como el nodo con más resultados obtenidos con distintos grupos taxonómicos.

\section{Consideraciones finales}

Se demuestra que los modelos digitales de elevación y el CAD resultan ser herramientas útiles para calcular el aumento de la distancia total de cada trazo individual al sobreponerse a la cartografía tridimensional; así como para calcular las distancias entre cada segmento de los trazos individuales y obtener distancias más cercanas a la realidad de las especies al tomarse en cuenta los valores de altitud en el modelo. Respecto a la influencia del relieve en la conformación de los trazos, se observó que, al menos en este caso, no modificó sustancialmente la 
topología de los trazos individuales de las 12 especies de roedores de la FVT. Esto puede deberse a tres razones principales: a) el par o tercia de puntos a medir se situaban dentro de la misma cota altitudinal; b) el registro más cercano en dos dimensiones siguió siendo el mismo sobre el MDE, ya que aun con el relieve los puntos tuvieron la misma proporción de distancia; c) se requieren curvas de nivel con una escala mayor (50 ó 100 metros) para obtener un MDE más detallado de la zona de estudio y calcular distancias aun más cercanas a las reales.

Dado que solo una especie (Pappogeomys bulleri) tuvo dos cambios en la topología de su trazo individual, es posible concluir que los análisis panbiogeográficos realizados hasta el momento permiten una buena aproximación a la realidad, incluyendo los métodos computarizados propuestos recientemente, aunque en adelante será necesario considerar la forma del relieve para generar hipótesis más adecuadas.

\section{Agradecimientos}

J. F. Parrot, V. Ochoa y R. Cartagena del Instituto de Geografía, brindaron apoyo en la elaboración de los modelos y análisis de los datos. J. F. Parrot proporcionó los programas para realizar algunos análisis. T. Escalante agradece las dos becas posdoctorales de DGAPA-UNAM (PROFIP 2004-2006, 2006-2007).

\section{Referencias bibliográficas}

ÁlVAREZ-MODRAGÓN, E. y MORRONE, J. J. Propuesta de áreas para la conservación de aves de México, empleando herramientas panbiogeográficas e índices de complementariedad. Interciencia, 2004, $\mathrm{N}^{\circ}$ 29, p. 112-120.

CAVALCANTI, M. J. Croizat: a software package for quantitative analysis in panbiogeography. Biogeografía, 2009, $N^{\circ} 4$, p. 4-6.

Figura $\mathrm{N}^{\circ} 4$

Trazos genealizados y nodo

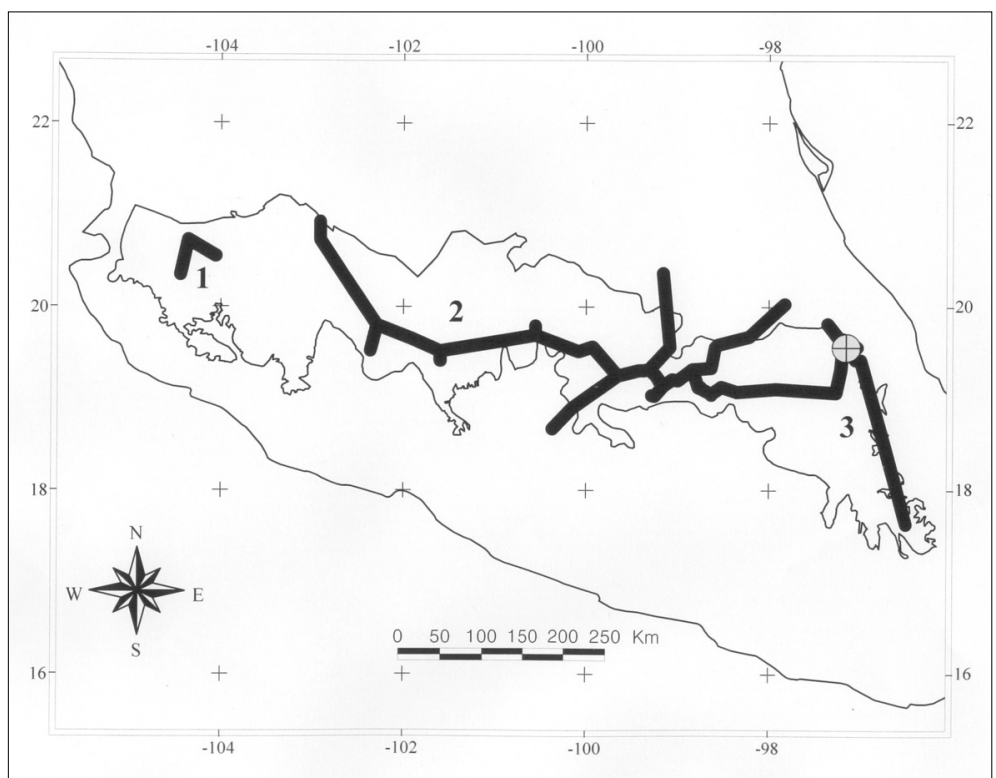

Fuente: Elaboración propia. 
CONTRERAS, R. y ELIOSA, H. Una visión panbiogeográfica preliminar de México. En: LLORENTE, J. y MORRONE, J. J. (eds.). Introducción a la biogeografía en Latinoamérica: teorías, conceptos, métodos y aplicaciones. Ciudad de México: UNAM, 2001, p. 197-219.

CROIZAT, L. Panbiogeography. Caracas: publicado por el autor, 1958.

CROIZAT, L. Space, time, form: the biological synthesis. Caracas: publicado por el autor, 1964.

CRAW, R. C.; GREHAN, J. R. \& HEADS, M. J. Panbiogeography, traking the history of life. Oxford: Oxford University Press, Biogeography Series N 11, 1999.

ESCALANTE, T.; ESPINOSA-ORGANISTA, D. y MORRONE, J. J. Patrones de distribución geográfica de los mamíferos terrestres de México. Acta Zoológica Mexicana (Nueva serie), 2002, $\mathrm{N}^{\circ} 87$, p. 47-65.

ESCALANTE, T.; RODRÍGuEZ，G. \& MORRONE, J. J. The diversification of Nearctic mammals in the Mexican Transition Zone. Biological Journal of the Linnean Society, 2004, №83, p. 327-339.

ESCALANTE, T.; RODRÍGUEZ, G. y MORRONE, J. J. Las provincias biogeográficas del componente Mexicano de Montaña desde la perspectiva de los mamíferos continentales. Revista Mexicana de Biodiversidad, 2005, № 76, p. 199-205.

ESCALANTE, T.; MORRONE, J. J. y RODRÍGUEZ, G. La distribución de los mamíferos terrestres y la regionalización biogeográfica natural de México. En: SÁNCHEZ-ROJAS, G. y ROJAS, A. (eds.). Tópicos en sistemática, biogeografía, ecología y conservación de mamíferos. Pachuca: Universidad Autónoma del Estado de Hidalgo, 2007a, p. 9-17.

ESCALANTE, T.; RODRÍGUEZ, G.; GÁMEZ, N.; LEÓN, L.; BARRERA, O. y SÁNCHEZ-CORDERO, V. Biogeografía y conservación de los mamíferos. En: LUNA, I. et al. (eds.). Biodiversidad de la Faja Volcánica
Transmexicana. Ciudad de México: UNAM, 2007b, p. 485-502.

ESRI. Arc View 3.2 GIS. Nueva York: Environmental Systems Research Institute, Inc., 1999.

ESRI. Arc Map 9.2, Arc GIS 9. Nueva York: Environmental Systems Research Institute, Inc., 2006.

FA, J. E. \& MORALES L. M. Mammals and protected areas in the Trans-Mexican Neovolcanic Belt. In: MARES, M. A. \& SCHMIDLY, D. J. (eds.). Latin American Mammalogy: history, biodiversity and conservation. Oklahoma: University of Oklahoma Press, Norman y London, 1991, p. 199-226.

FELICÍSIMO, A. M. Modelos Digitales de Terreno. Introducción y aplicaciones en las ciencias ambientales. Oviedo: Ed. Pentalfa, 1994.

FELICÍsIMO, A. M. La utilización de los MDT en los estudios del medio físico. Extremadura: Universidad de Extremadura, 1999.

FERRUSQUÍA-VILLAFRANCA， I. Geología de México: una sinopsis. En: RAMAMOORTHY, T. P. et al. (eds.). Diversidad biológica de México: Orígenes y distribución. Ciudad de México: Instituto de Biología, UNAM, 1993, p. 3-108.

GARCÍA-ESTRADA， G. y LÓPEZHERNÁNDEZ, A. Modelos digitales de elevación del terreno: uso en la geología estructural. Notas. Revista de Información y Análisis, 2003, № 22, p. 77-87.

GONZÁLEZ-MORENO, J. O. El papel de la pendiente del terreno en la estimación precisa de superficies para los recursos naturales: el caso de los recursos forestales. Tesis licenciatura en Geografía. Ciudad de México: Facultad de Filosofía y Letras, Universidad Nacional Autónoma de México, México, 2003.

GREHAN, J. R. Panbiogeografía y la geografía de la vida. En: LLORENTE, J. y 
MORRONE, J. J. (eds.). Introducción a la biogeografía en Latinoamérica: teorías, conceptos, métodos y aplicaciones. Ciudad de México: UNAM, 2001, p. 181-195.

JASC, Inc. Paint Shop Pro 4.12. Minneapolis: JASC Software, 1996.

LIRIA, J. Sistemas de información geográfica y análisis espaciales: un método combinado para realizar estudios panbiogeográficos. Revista Mexicana de Biodiversidad, 2008, N 79, p. 281-284.

MORRONE, J. J. Panbiogeografía, componentes bióticos y zonas de transición. Revista Brasileira de Entomologia, 2004, Nº 48, p. 149-162.

MORRONE, J. J. Hacia una síntesis biogeográfica de México. Revista Mexicana de Biodiversidad, 2005, N 76, p. 207-252.

MORRONE, J. J. Hacia una biogeografía evolutiva. Revista Chilena de Historia Natural, 2007, Nº 80, p. 509-520.

MORRONE, J. J. Evolutionary biogeography: An integrative approach with case studies. Nueva York: Columbia University Press, 2009.

MUNGUÍA, C. M. Representatividad mastofaunística en Áreas Naturales Protegidas y Regiones Terrestres Prioritarias en el Eje
Neovolcánico: un modelo de conservación. Tesis Licenciatura en Biología. Ciudad de México: Facultad de Ciencias, Universidad Nacional Autónoma de México, México, 2004.

RAMÍREZ-PULIDO, J.; ARROYOCABRALES, J. y CASTRO-CAMPILLO, A. Estado actual y relación nomenclatural de los mamíferos terrestres de México. Acta Zoológica Mexicana (Nueva serie), 2005, Nº 21, p. 21-82.

ROJAS, C. A. Automatización del método de la Panbiogeografía: identificación de centros de diversidad del Parque Nacional Iztaccíhuatl, Popocatépetl, Zoquiapan y anexas. Tesis Maestría. Ciudad de México: Universidad Nacional Autónoma de México, México, 2004.

ROJAS, C. A. Una herramienta automatizada para realizar análisis panbiogeográficos. Biogeografía, 2007, № 1, p. 31-33.

TORRES-MIRANDA, A. y LUNA-VEGA, I. Análisis de trazos para establecer áreas de conservación en la Faja Volcánica Transmexicana. Interciencia, 2006, № 31, p. 849-855.

VILLA, B. y CERVANTES, F. A. LOS mamíferos de México. Ciudad de México: Grupo Editorial Iberoamericana S. A. de C. V., 2003. 